10. Molofsky, A. V. et al. Bmil dependence distinguishes neural stem cell self-renewal from progenitor proliferation. Nature 425, 962-967 (2003)

11. Wechsler-Reya, R. J. \& Scott, M. P. Control of neuronal precursor proliferation in the cerebellum by Sonic Hedgehog. Neuron 22, 103-114 (1999).

12. Rubin, J. B. \& Rowitch, D. H. Medulloblastoma: a problem of developmental biology. Cancer Cell 2 , 7-8 (2002).

13. Zurawel, R. H. et al. Analysis of PTCH/SMO/SHH pathway genes in medulloblastoma. Genes Chromosom. Cancer 27, 44-51 (2000).

14. Wechsler-Reya, R. \& Scott, M. P. The developmental biology of brain tumors. Annu. Rev. Neurosci. 24, 385-428 (2001).

15. Taylor, M. D. et al. Mutations in SUFU predispose to medulloblastoma. Nature Genet. 31, 306-310 (2002)

16. Dong, J., Gailani, M. R., Pomeroy, S. L., Reardon, D. \& Bale, A. E. Identification of PATCHED mutations in medulloblastomas by direct sequencing. Hum. Mutat. 16, 89-90 (2000).

17. Pietsch, T. et al. Medulloblastomas of the desmoplastic variant carry mutations of the human homologue of Drosophila patched. Cancer Res. 57, 2085-2088 (1997).

18. Pomeroy, S. L. et al. Prediction of central nervous system embryonal tumour outcome based on gene expression. Nature 415, 436-442 (2002)

19. Herms, J. et al. C-MYC expression in medulloblastoma and its prognostic value. Int. J. Cancer 89, 395-402 (2000)

20. Tomlinson, F. H. et al. Aggressive medulloblastoma with high-level N-myc amplification. Mayo Clin. Proc. 69, 359-365 (1994).

21. Marino, S., Vooijs, M., van Der Gulden, H., Jonkers, J. \& Berns, A. Induction of medulloblastomas in $\mathrm{p} 53$-null mutant mice by somatic inactivation of $\mathrm{Rb}$ in the external granular layer cells of the cerebellum. Genes Dev. 14, 994-1004 (2000).

22. Lee, Y. et al. A molecular fingerprint for medulloblastoma. Cancer Res. 63, 5428-5437 (2003).

23. Smith, K. S. et al. Bmi- 1 regulation of INK4A-ARF is a downstream requirement for transformation of hematopoietic progenitors by E2a-Pbxl. Mol. Cell 12, 393-400 (2003).

24. Ruppert, J. M., Vogelstein, B. \& Kinzler, K. W. The zinc finger protein GLI transforms primary cells in cooperation with adenovirus E1A. Mol. Cell. Biol. 11, 1724-1728 (1991).

25. Miyoshi, H., Blomer, U., Takahashi, M., Gage, F. H. \& Verma, I. M. Development of a self-inactivating lentivirus vector. J. Virol. 72, 8150-8157 (1998).

Supplementary Information accompanies the paper on www.nature.com/nature.

Acknowledgements We thank P. Kleihues and H. Ohgaki for providing medulloblastoma samples; M. Grotzer for medulloblastoma cell lines; E. R. Fearon for RK3E cells; M. Ruppert for Gli cDNAs; D. Trono for the lentivirus Bmil construct; I. Camenisch for technical help; and K. Kieboom for animal care. MATH-1 and mGluR-2 probes were gifts from $\mathrm{H}$. Zoghbi and S. Nakanishi, respectively. We thank A. Lund, M. Hernandez and S. Bruggeman for discussions, and P. U. Heitz for support. This work was supported by grants from the 'Krebsforschung Schweiz' to S.M. and from the 'Novartis Stiftung' to S.M.; M. L. and E. T. were supported by a Pioneer grant from the Netherlands organization for Scientific Research to M.v.L

Competing interests statement The authors declare that they have no competing financial interests.

Correspondence and requests for materials should be addressed to S.M (silvia.marino@usz.ch) or M.v.L (m.v.lohuizen@nki.nl).

\section{The protein kinase PKR is required for macrophage apoptosis after activation of Toll-like receptor 4}

Li-Chung Hsu ${ }^{1}$, Jin Mo Park ${ }^{1}$, Kezhong Zhang ${ }^{3}$, Jun-Li Luo ${ }^{1}$, Shin Maeda ${ }^{1}$, Randal J. Kaufman ${ }^{3}$, Lars Eckmann ${ }^{2}$, Donald G. Guiney ${ }^{2}$ \& Michael Karin ${ }^{1}$

${ }^{1}$ Laboratory of Gene Regulation and Signal Transduction, Department of Pharmacology, and ${ }^{2}$ Department of Medicine, School of Medicine, University of California, San Diego, 9500 Gilman Drive, La Jolla, California 92093-0636, USA ${ }^{3}$ Howard Hughes Medical Institute, Department of Biological Chemistry, University of Michigan, 1150 W. Medical Center Drive, Ann Arbor, Michigan 48109-0659, USA

Macrophages are pivotal constituents of the innate immune system, vital for recognition and elimination of microbial pathogens $^{1}$. Macrophages use Toll-like receptors (TLRs) to detect pathogen-associated molecular patterns-including bacterial cell wall components, such as lipopolysaccharide or lipoteichoic acid, and viral nucleic acids, such as double-stranded (ds)RNAand in turn activate effector functions, including anti-apoptotic signalling pathways ${ }^{2}$. Certain pathogens, however, such as Salmonella spp., Shigellae spp. and Yersiniae spp., use specialized virulence factors to overcome these protective responses and induce macrophage apoptosis ${ }^{3}$. We found that the anthrax bacterium, Bacillus anthracis, selectively induces apoptosis of activated macrophages ${ }^{4}$ through its lethal toxin, which prevents activation of the anti-apoptotic $\mathrm{p} 38$ mitogen-activated protein kinase ${ }^{4}$. We now demonstrate that macrophage apoptosis by three different bacterial pathogens depends on activation of TLR4. Dissection of anti- and pro-apoptotic signalling events triggered by TLR4 identified the dsRNA responsive protein kinase PKR as a critical mediator of pathogen-induced macrophage apoptosis. The pro-apoptotic actions of PKR are mediated both through inhibition of protein synthesis and activation of interferon response factor 3.

At least ten TLRs are known, and some of the pathogenassociated molecular patterns that cause their activation were identified $^{2}$. Lethal toxin (LT) or p38 inhibitors induce apoptosis in macrophages incubated with either lipopolysaccharide (LPS) or lipoteichoic acid (LTA), derived from Gram-negative and Grampositive bacteria, respectively ${ }^{4}$. We have now found that heatinactivated B. anthracis, a Gram-positive bacterium, induces extensive macrophage apoptosis in the presence of SB202190, a p38 inhibitor, but bone-marrow-derived macrophages (BMDMs) from $\mathrm{C} 3 \mathrm{H} / \mathrm{HeJ}$ mice, whose TLR4 is inactive ${ }^{5}$, are resistant to such killing (Fig. 1a). The apoptotic response to heat-killed B. anthracis was also seen with bacteria grown in LPS-free medium (data not shown). In wild-type BMDMs, a strong apoptotic response dependent on p38 inhibition was detected only upon treatment with the TLR4 agonist, LPS (Fig. 1b). Little or no apoptosis was seen after incubation with the TLR2 agonist synthetic bacterial lipopeptide $\left(\mathrm{Pam}_{3} \mathrm{CSK}_{4}\right)$ or the TLR9 agonist immunostimulatory (CpG-containing) DNA. The TLR3 agonist, synthetic dsRNA_poly(IC)_induced a weak apoptotic response even without p38 inhibition. Transient expression of a CD4-hToll chimaera ${ }^{6}$, in which the intracellular Toll-IL-1 receptor (TIR) domain of TLR4 was fused to the extracellular and transmembrane domains of CD4, also resulted in apoptosis after p38 inhibition (Fig. 1c). Consistent with the critical role of TLR4, BMDMs from $\mathrm{C} 3 \mathrm{H} / \mathrm{HeJ}$ mice, but not from the equivalent wildtype strain, $\mathrm{C} 3 \mathrm{H} / \mathrm{HeOuJ}$, were resistant to apoptosis induced by LPS plus SB202190 (Fig. 1d).

TLR4 uses several adaptor proteins, including MyD88, MAL/ TIRAP, TRIF and TRAM to engage downstream signalling proteins and eventually activate ІкB kinase (IKK) and mitogen-activated protein kinases (MAPKs) ${ }^{7,8}$ (Fig. 2a). Macrophages from mice lacking MyD88 (ref. 9) or TRAF6, a signalling protein that acts downstream of MyD88 and TIRAP ${ }^{10}$, still undergo apoptosis after LPS stimulation and p38 inhibition (Fig. 2b, c). In fact, both $\mathrm{MyD}^{-1-}$ and $\mathrm{TRAF}^{-1-}$ macrophages exhibit an increased apoptotic response. NF-кB activation and ІкB degradation, which depend on IKK $\beta^{11}$, are reduced in both MyD88 $8^{-/-}$and TRAF6 $^{-/-}$ macrophages (Fig. $2 \mathrm{~d}$ and data not shown). As NF- $\mathrm{kB}$ activates antiapoptotic genes ${ }^{11}$, these defects may explain the enhanced apoptosis of MyD $88^{-1-}$ and TRAF6 ${ }^{-l-}$ macrophages. Indeed, IKK $\beta$-deficient macrophages generated by crossing $I k k \beta^{F / F}$ mice ${ }^{12}$ with mice expressing Cre recombinase from the IFN-inducible MX1 promoter $^{13}$, were defective in NF-кB activation (data not shown) and underwent apoptosis upon incubation with LPS, LTA or TNF- $\alpha$, even without p38 inhibition (Fig. 2e). IKK $\beta$-deficient macrophages were also more susceptible to poly(IC)-induced apoptosis. Deletion of IKK $\beta$ in macrophages did not affect p38 expression (Fig. 2e) or activation (data not shown).

Although TLR4 activation results in TNF- $\alpha$ production, the apoptosis observed in TLR4-activated and p38-inhibited macrophages was not prevented by ablating type I TNF- $\alpha$ receptor (unpublished results). Furthermore, unlike apoptosis induced by TNF- $\alpha$, caspase- 8 was not activated during LPS-induced apoptosis 
(Supplementary Fig. 1a). Yet, cleavage of caspases 3, 6, 7 and 9 and cytochrome $c$ release were readily observed (Supplementary Fig. 1b; data not shown), and apoptosis was inhibited by a pan-caspase inhibitor (Supplementary Fig. 1c). Hence, inhibition of p38 or IKK unleashes the ability of TLR4 to deliver a cell death signal through the mitochondrial-dependent pathway ${ }^{11}$.

Another protein involved in TLR signalling is the dsRNAresponsive kinase $\mathrm{PKR}^{14}$. $\mathrm{PKR}$ was suggested to mediate apoptosis in fibroblasts in response to viral infection and inflammatory cytokines $^{15}$. However, PKR also activates IKK and NF- $\mathrm{B}^{16}$ and thereby suppresses apoptosis. To determine the role of PKR in macrophage apoptosis, we examined its regulation and found it to be rapidly activated by either LPS or poly(IC) (Fig. 3a). Activation of PKR by either stimulus depended on TRIF (Fig. 3b). Ablation of PKR did not affect p38 or IKK activation in response to LPS (Fig. 3c) and with the exception of decreased IFN $\beta$ (Fig. 3d) or inducible NO synthase (iNOS; Fig. 3e) expression, $\mathrm{PKR}^{-1-}$ BMDMs did not exhibit reduced induction of numerous NF- $\mathrm{KB}$ target genes, including those coding for anti-apoptotic proteins, such as c-IAP2, c-FLIP, A1a, A20, and Gadd45 $\beta$ (Fig. 3d). $\mathrm{PKR}^{-1-}$ BMDMs also exhibited
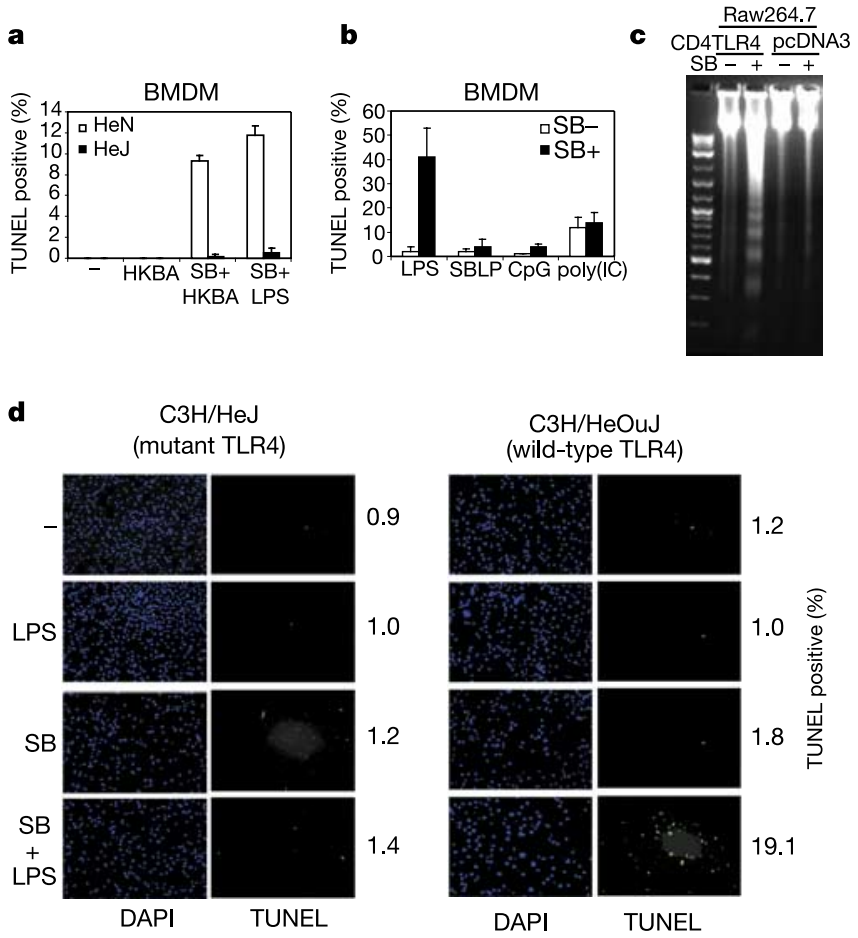

Figure 1 Heat-killed $B$. anthracis (HKBA) and LPS induce macrophage apoptosis through TLR4. a, HKBA induces macrophage apoptosis in a TLR4-dependent manner. BMDMs from $\mathrm{C} 3 \mathrm{H} / \mathrm{HeN}$ (TIr4 wild-type) or C3H/HeJ (T/r 4 mutant) mice were incubated with HKBA or LPS (100 $\mathrm{ng} \mathrm{ml}^{-1}$ ) with or without p38 inhibitor (SB202190, $\left.10 \mu \mathrm{M}\right)$. After 18 h, apoptosis was scored by TUNEL staining. Results in this and all similar experiments were repeated several times and one representative done in triplicates is shown. Values represent averages \pm s.d. $\mathbf{b}$, TLR4 agonists induce apoptosis in the presence of a p38 inhibitor. C57BL/6 BMDMs were treated with different TLR agonists: LPS, synthetic bacterial lipopeptide $\left(1 \mu \mathrm{g} \mathrm{ml}^{-1}\right)$, synthetic CpG-containing DNA $(1 \mu \mathrm{M})$, or poly(IC) $\left(10 \mu \mathrm{g} \mathrm{ml}^{-1}\right)$, with or without SB202190. c, TLR4 cytoplasmic domain transduces an apoptotic signal. RAW264.7 cells were transfected with a vector encoding a CD4-hTLR4 fusion protein, or an empty vector (pcDNA3). After $24 \mathrm{~h}$, transfectants were incubated with or without SB202190, genomic DNA was isolated after $18 \mathrm{~h}$ and analysed by agarose gel electrophoresis for a nucleosomal ladder indicative of apoptosis. d, TLR4 is required for induction of apoptosis. $\mathrm{C} 3 \mathrm{H} / \mathrm{HeJ}$ or $\mathrm{C} 3 \mathrm{H} / \mathrm{HeO} \mathrm{UJ} \mathrm{BMDMs}$ were incubated with or without LPS in the presence or absence of SB202190 for $18 \mathrm{~h}$ and analysed by DAPI (blue) and TUNEL (green) staining. The percentage of TUNEL-positive cells is shown on the right. defective STAT1 phosphorylation in response to LPS (Fig. 3f), which depends on autocrine production of type I IFNs because it was not observed in macrophages deficient in type I IFN receptor (IFNRI) (Supplementary Fig. 2).

Most importantly, $\mathrm{PKR}^{-{ }^{-}}$macrophages did not undergo apop-

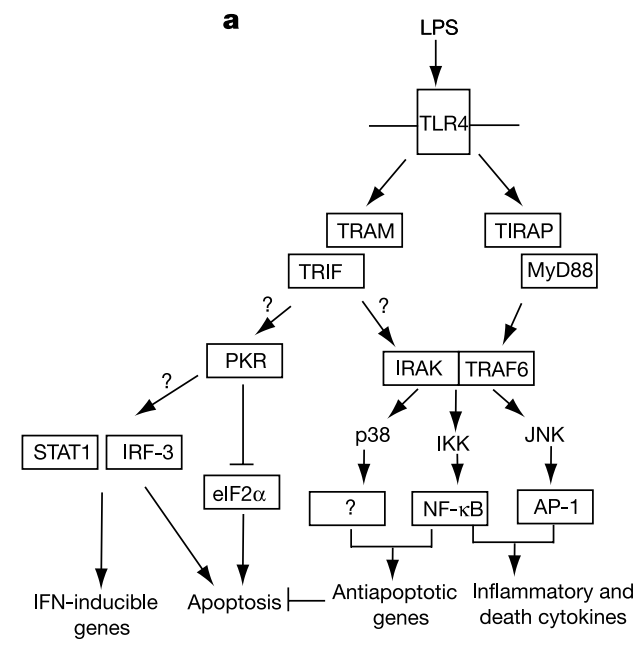

b

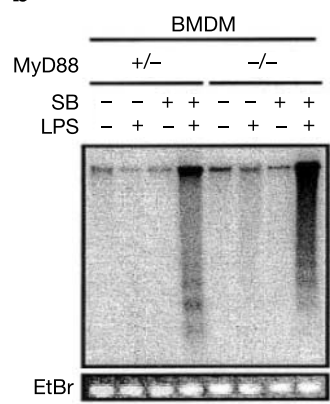

c

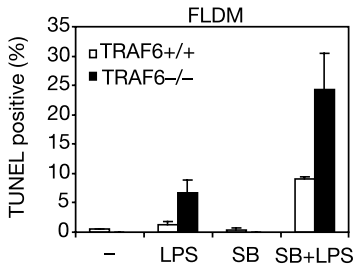

d

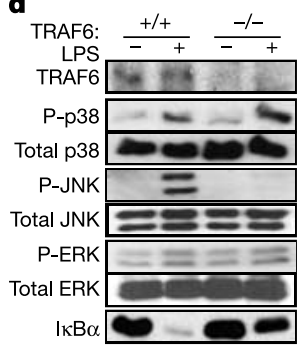

e

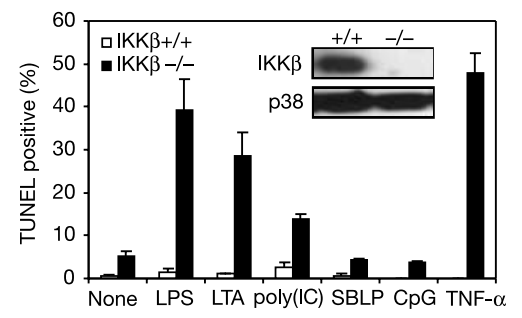

Figure 2 Role of effector molecules in TLR4-induced apoptosis. a, A diagram of currently known TLR4-stimulated signalling pathways in macrophages. Question marks denote yet-to-be established connections. b, Role of MyD88. MyD88 ${ }^{+/-}$and MyD88 ${ }^{-1-}$ BMDMs were incubated with or without LPS in the presence or absence of SB202190. After $18 \mathrm{~h}$, genomic DNA was isolated and end-labelled with $\left[\alpha-\mathrm{P}^{32}\right]-\mathrm{dATP}$ and Taq polymerase, followed by agarose gel electrophoresis and autoradiography. Gel loading was examined by EtBr staining. c, Role of TRAF6. Fetal-liver-derived TRAF6 ${ }^{+/+}$and $\mathrm{TRAFG}^{-/-}$macrophages (FLDMs) were stimulated as above, and apoptotic cell death was quantified by TUNEL staining. $\mathbf{d}$, Characterization of LPS signalling. TRAF6 ${ }^{+/+}$and

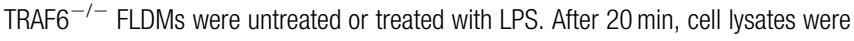
prepared and analysed by immunoblotting with antibodies specific to TRAF6, IкB $\alpha$, different MAPKs and their phosphorylated forms. e, Role of IKK 3 . BMDMs from Ikk $\mathrm{F} / \mathrm{F}$ $\left(\mathrm{IKK} \beta^{+/+}\right)$or MX1Cre-lkK$\beta^{\mathrm{F} / \mathrm{F}}\left(\mathrm{IKK} \beta^{-/-}\right)$mice were untreated or treated with LPS, LTA from B. subtilis $\left(10 \mu \mathrm{g} \mathrm{ml}^{-1}\right)$, poly(IC) $\left(10 \mu \mathrm{g} \mathrm{ml}^{-1}\right)$, synthetic bacterial lipopeptide, SBLP $\left(1 \mu \mathrm{gl}^{-1}\right)$, CpG-containing DNA $(1 \mu \mathrm{M})$, or mouse TNF- $\alpha\left(10 \mathrm{ng} \mathrm{m}^{-1}\right)$. After $12 \mathrm{~h}$, apoptotic cell death was quantified by TUNEL staining. Inset, macrophage lysates were analysed by immunoblotting with antibodies specific to IKK $\beta$ and p38. 
tosis upon incubation with LPS and either SB202190 or LT (Fig. 4a) or after prolonged incubation with LPS alone (Supplementary Fig. 3a). $\mathrm{PKR}^{-1-}$ macrophages were, however, fully sensitive to other pro-apoptotic stimuli, such as doxorubicin or 15-deoxy$\Delta^{12,14}$ prostaglandin $\mathrm{J}_{2}$ (Supplementary Fig. 3b). PKR activation contributes to induction of type I IFNs, such as IFN $\beta$, which can further increase its expression ${ }^{17}$. Type I IFNs sensitize myeloid cells to LPS, LTA or bacterial-induced apoptosis ${ }^{18}$. Whereas IFN $\beta$ alone did not induce apoptosis in BMDMs, it rendered them susceptible to LPS-induced apoptosis even without SB202190 (Fig. 4b). IFN $\beta$ also potentiated the apoptosis of wild-type BMDMs exposed to LPS + SB202190, but PKR $^{-1-}$ BMDMs were resistant to these effects of IFN $\beta$ (Fig. 4b). Nevertheless, BMDMs from IFNRI $^{-/-}$

a

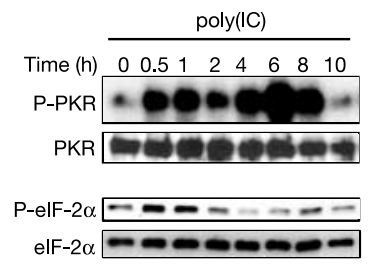

b

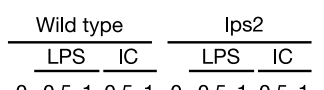

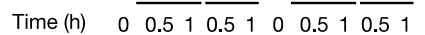

P-PKR $\approx \mathbf{0} \mathbf{0}$

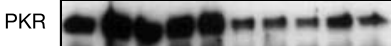

PKR

$\beta$-actin

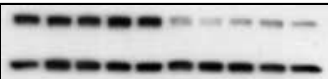

d

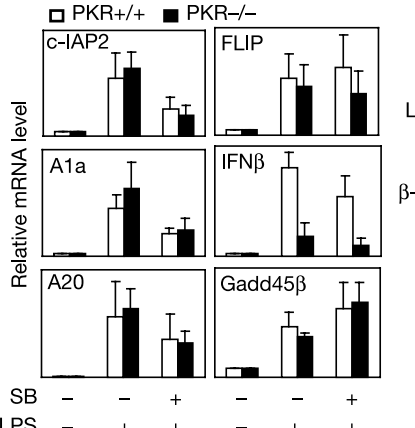

LPS

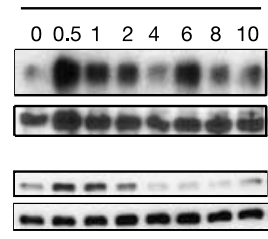

c

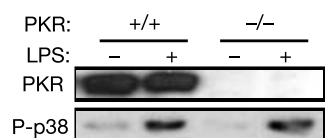

$\mathrm{p} 38 \longrightarrow$

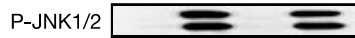

JNK $1 / 2 \Longrightarrow$

P-ERK $\approx \cong \approx$

ERK 23

$\mathrm{P}-\mathrm{I} \mathrm{B} \mathrm{B} \alpha+\mathrm{cos}$

$\mathrm{I \kappa B} \alpha \longrightarrow$

e $\mathrm{PKR}+/+\quad \mathrm{PKR}-/-$

LPS (h) $\overline{024681012} \overline{024681012}$

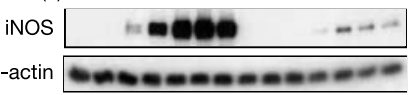

f

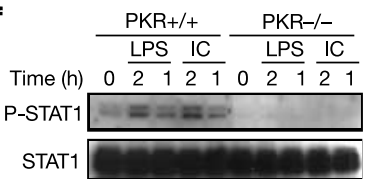

P-p38 $=$

Figure 3 PKR is necessary for LPS-induced interferon signalling pathway in macrophages. a, Activation of PKR by LPS. BMDMs were stimulated with LPS (100 $\left.\mathrm{ng} \mathrm{ml}^{-1}\right)$ or poly $(\mathrm{IC})\left(10 \mu \mathrm{g} \mathrm{m}^{-1}\right)$. At the indicated time points cells were lysed and PKR activation was monitored by autophosphorylation. Gel loading was controlled by immunoblotting for PKR. The lysates were monitored for elF-2 $\alpha$ phosphorylation by immunoblotting with antibodies specific for phosphorylated elF-2 $\alpha$ (P-elF-2 $\alpha$ ) and total elF-2 $\alpha$. b, PKR acts downstream of TRIF. Wild-type and Ips2 (TRIF-deficient) BMDMs were stimulated with LPS or poly(IC). PKR activation was monitored by

autophosphorylation. The same lysates were examined for PKR and $\beta$-actin content by immunoblotting. c, Normal MAPK and IKK activation in PKR ${ }^{-/-}$macrophages. PKR ${ }^{+/+}$ and $\mathrm{PKR}^{-/-}$BMDMs were left unstimulated or stimulated with LPS. After $20 \mathrm{~min}$, cell lysates were prepared and immunoblotted with antibodies to different MAPKs or $I_{\kappa} B \alpha$ and their phosphorylated forms. $\mathbf{d}$, PKR is required for IFN $\beta$ induction but is dispensable for induction of anti-apoptotic genes. $\mathrm{PKR}^{+/+}$and $\mathrm{PKR}^{-/-}$BMDMs were incubated with or without LPS in the absence or presence of SB202190. After $4 \mathrm{~h}$, total cellular RNA was isolated, and relative gene expression was determined by real-time PCR. The results are averages of three separate experiments normalized to the level of cyclophilin mRNA.

e, f, PKR is required for iNOS induction (e) and STAT1 phosphorylation (f). PKR ${ }^{+/+}$and $\mathrm{PKR}^{-1-}$ BMDMs were incubated with LPS or poly(IC). At the indicated time points, cell lysates were prepared and iNOS expression and STAT1 phosphorylation were examined by immunoblotting. mice $^{19}$ still underwent apoptosis when incubated with LPS and SB202190 (Fig. 4c) and exhibited normal PKR activation by LPS (Supplementary Fig. 4). Thus, although PKR contributes to IFN $\beta$ production, which can potentiate macrophage apoptosis, IFN $\beta$ signalling itself is not essential for TLR4-induced PKR activation or macrophage apoptosis.

PKR is activated by poly(IC), which induces macrophage apoptosis even without p38 inhibition (Fig. 1b). Activated PKR phosphorylates eukaryotic translation initiation factor $2 \alpha(\operatorname{eIF} 2 \alpha)$ at serine 51 and thereby inhibits protein synthesis ${ }^{20}$. Although the kinase activity of PKR is not required for NF- $\mathrm{kB}$ activation, it is needed for induction of apoptosis (Supplementary Fig. 5). In addition, macrophages are very sensitive to protein synthesis inhibition (Supplementary Fig. 6). Hence, PKR activation may cause macrophage apoptosis by inhibiting synthesis of anti-apop-
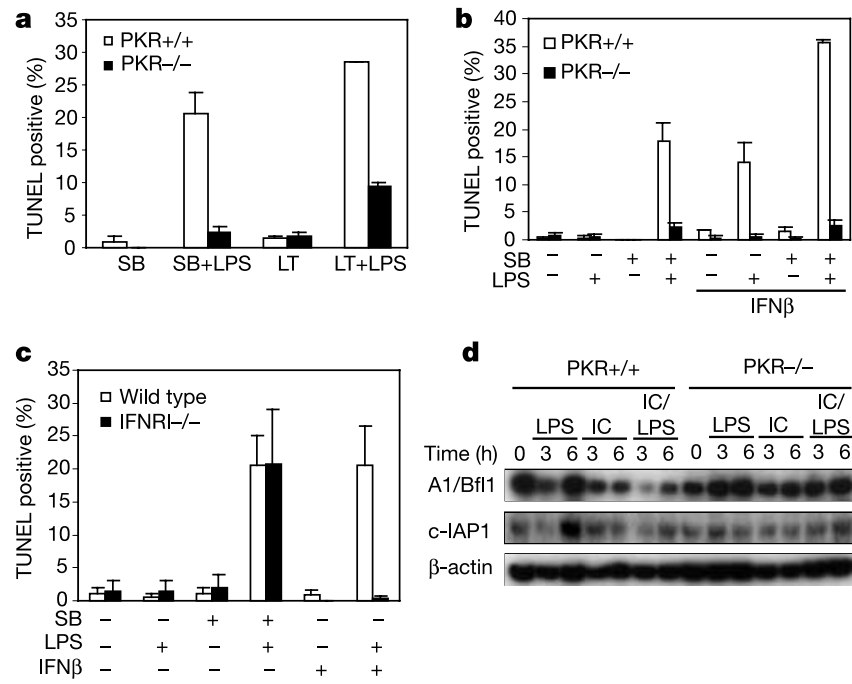

d

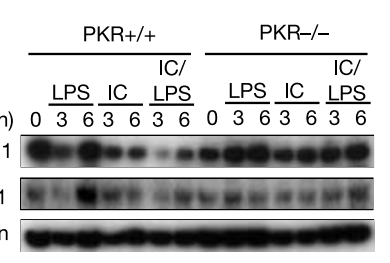

e
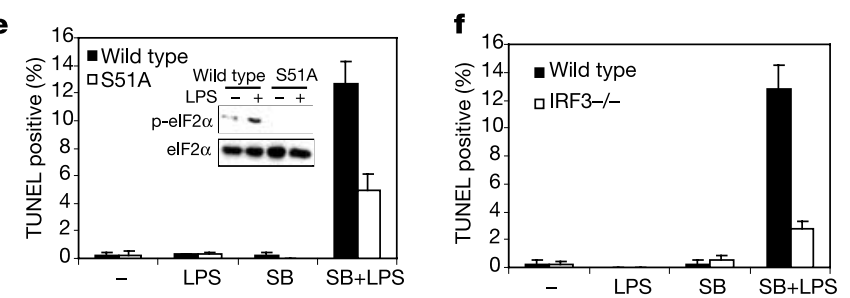

Figure 4 PKR is required for LPS-induced macrophage apoptosis. a, PKR-deficient BMDMs are resistant to LPS-induced apoptosis. $\mathrm{PKR}^{+/+}$and $\mathrm{PKR}^{-/-}$BMDMs were left unstimulated or stimulated with either LPS in the presence or absence of either SB202190 or LT (500 $\mathrm{ng} \mathrm{ml}^{-1} \mathrm{LF}$ and $2.5 \mu \mathrm{g} \mathrm{ml}^{-1}$ protective antigen, PA), and the extent of apoptosis was determined after $18 \mathrm{~h}$. b. IFN $\beta$ sensitizes BMDMs to LPS-induced apoptosis. PKR ${ }^{+/+}$and PKR ${ }^{-/-}$BMDMs were incubated with or without LPS in the absence or presence of SB202190 or IFN $\beta\left(1,000 \mathrm{U} \mathrm{ml}^{-1}\right)$ for $18 \mathrm{~h}$ and the extent of apoptosis was determined. c, Type I IFN signalling is not required for LPS-induced macrophage apoptosis. BMDMs from IFNR $1^{+/+}$or IFNR $1^{-/-}$mice were incubated with or without LPS in the presence or absence of SB202190 and IFN $\beta$ for $18 \mathrm{~h}$ and the extent of apoptosis was determined. $\mathbf{d}$, PKR activation inhibits $A 1 / B f 1$ expression. PKR ${ }^{+/+}$and $\mathrm{PKR}^{-1-}$ BMDMs were transfected with or without poly(IC) using Lipofectamine. After $6 \mathrm{~h}$, LPS was added and the levels of A1/Bfl1 and clAP-1 were examined by immunoblotting. e, elF2 $\alpha$ phosphorylation is required for induction of macrophage apoptosis. BMDMs derived from lethally irradiated mice reconstituted with fetal liver stem cells from either wild-type or elF2 $\alpha(S 51 A)$ mice ${ }^{22}$ were incubated with LPS with or without SB202190 and the extent of apoptosis was analysed. The inset shows the absence of elF2 $\alpha$ phosphorylation in knockin macrophages. $\mathbf{f}$, IRF3 is required for induction of macrophage apoptosis. BMDMs from wild-type or IRF3 ${ }^{-1-}$ mice were incubated with or without LPS in the presence or absence of SB202190 for $18 \mathrm{~h}$ and the extent of apoptosis was determined. 
totic proteins. To enhance PKR activation and bypass TLR3, which may activate anti-apoptotic pathways, we introduced poly(IC) into macrophages by transfection ${ }^{21}$. This resulted in higher levels of PKR activity and caused a higher level of apoptosis (data not shown). Transfection of wild-type BMDMs with poly(IC) followed by LPS treatment inhibited accumulation of A1/Bfl1, an anti-apoptotic member of the Bcl 2 family known to inhibit LPS-induced apoptosis of neutrophils ${ }^{11}$, and to a lesser extent c-IAP1. The same treatment of $\mathrm{PKR}^{-1-}$ BMDMs did not reduce the level of either protein (Fig. 4d).

To examine directly the role of eIF $2 \alpha$ phosphorylation in macrophage apoptosis, we transplanted fetal liver haematopoietic progenitors from $e I F 2 \alpha(S 51 A)$ knockin mice, which die shortly after birth, to lethally irradiated wild-type mice, in order to obtain macrophages that express an eIF $2 \alpha$ variant in which serine 51 , the major PKR phosphorylation site, was replaced with an alanine ${ }^{22}$. eIF2 $\alpha$ (S51A) BMDMs were considerably less sensitive than wildtype BMDMs to apoptosis caused by incubation with LPS and SB202190 (Fig. 4f). The residual apoptotic response in $e I F 2 \alpha(S 51 A)$ macrophages suggested the existence of another PKR-dependent pro-apoptotic pathway. It was proposed that interferon response factor 3 (IRF3), a transcription factor activated by dsRNA, is an important mediator of virus-induced apoptosis ${ }^{23}$. We found that BMDMs from IRF3 ${ }^{-1-}$ mice $^{24}$ showed increased resistance to LPS + SB202190 (Fig. 4f).

The role of TLR4 and PKR in apoptosis induced by live pathogenic bacteria was investigated using BMDMs infected with B. anthracis, Yersinia and Salmonella. $\mathrm{PKR}^{-1-}$ macrophages showed markedly reduced levels of apoptosis compared with $\mathrm{PKR}^{+/+}$cells (Fig. 5a, b). The result with live anthrax bacilli was similar to the one obtained with LT regarding the PKR dependence of apoptosis, but did not require addition of LPS (compare Fig. 5a to Fig. 4a). Pathogens that induce macrophage apoptosis activate TLR4 through cell wall components, but apoptosis also requires a specific contribution from the bacteria. Yersinia spp. injects YopJ, an inhibitor of MAPK and IKK activation ${ }^{25}$, into the host cell cyto- plasm. As expected, a yopJ mutant of Yersinia pseudotuberculosis did not induce apoptosis in $\mathrm{PKR}^{+/+}$macrophages (data not shown).

For Salmonella infections, PKR-dependent macrophage apoptosis requires the SPI2 locus (data not shown), which is responsible for translocation of bacterial virulence proteins from the phagosome into the macrophage cytoplasm ${ }^{26}$. However, the Salmonella SipB protein, a caspase 1 activator ${ }^{26}$, was dispensable for PKR-dependent apoptosis (data not shown), a finding consistent with the distinct mechanism of SipB-mediated cell death that differs from classical apoptosis $^{26}$. Consistent with the results described above, eIF2(S51A) and IRF ${ }^{-1-}$ macrophages were also less susceptible to Salmonellainduced apoptosis (Fig. 5c, d). BMDMs from TLR4-deficient mice exhibited a dramatically reduced apoptotic response after infection with B. anthracis (Supplementary Fig. 7) and as shown in Fig. 1a, the Tlr4 mutation in the $\mathrm{C} 3 \mathrm{H} / \mathrm{HeJ}$ strain prevented macrophage apoptosis by heat-killed B. anthracis. The apoptotic response to Salmonella and Yersinia was also considerably reduced in BMDMs from $\mathrm{C} 3 \mathrm{H} / \mathrm{HeJ}$ mice (Fig. 5e). Thus, the TLR4 to PKR pathway is crucial for macrophage apoptosis elicited by both Gram-positive and Gram-negative pathogens.

Microbial-induced macrophage apoptosis may represent a major mechanism allowing pathogenic bacteria to avoid detection and destruction by the innate immune system ${ }^{3}$. Virulence factors used by certain pathogens to dismantle host defences were identified and in some cases shown to act through inhibition of anti-apoptotic signalling pathways $s^{4,25,26}$. The results described above shed further light on this phenomenon and identify what appears to be a general mechanism used by three different bacterial pathogens, B. anthracis, Yersinia and Salmonella, to specifically kill activated macrophages. We find that macrophage apoptosis by either Gram-positive (B. anthracis) or Gram-negative (Yersinia, Salmonella) pathogens requires activation via TLR4. Curiously, however, TLR4 is not a typical death receptor with death domains that cause caspase- 8 activation. In fact, TLR4 engagement results in activation of both anti-apoptotic and pro-apoptotic signalling pathways. Normally, the anti-apoptotic pathways, which depend on MyD88 and TIRAP/

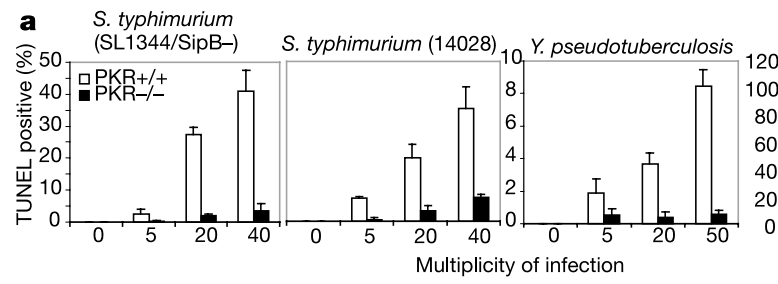

c

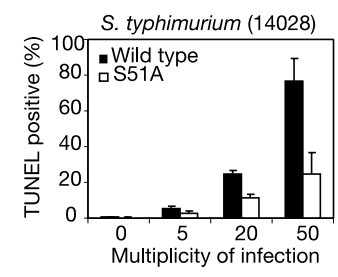

e
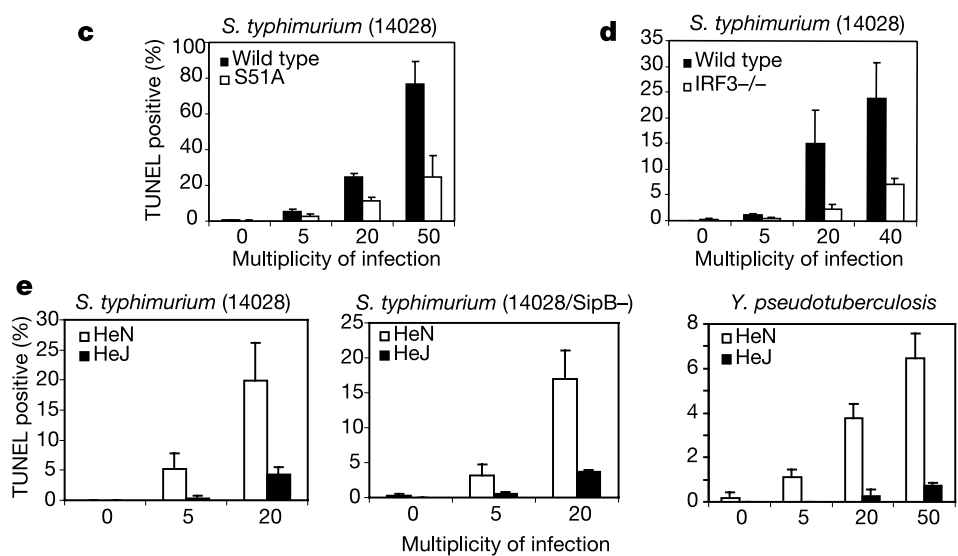

\section{b}

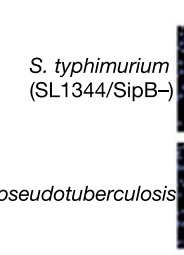

B. anthracis

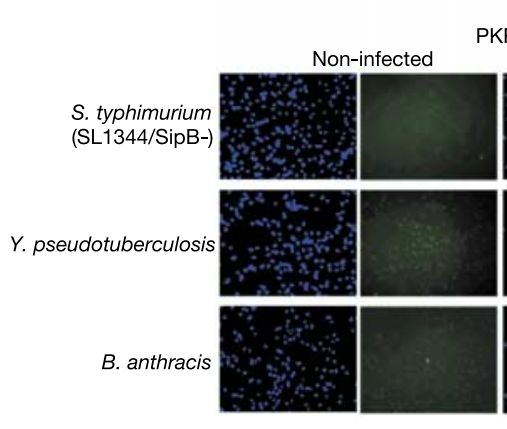

$\mathrm{PKR}+/+$
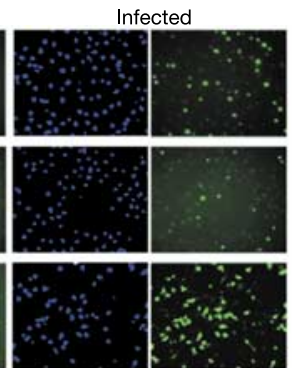

PKR-/-

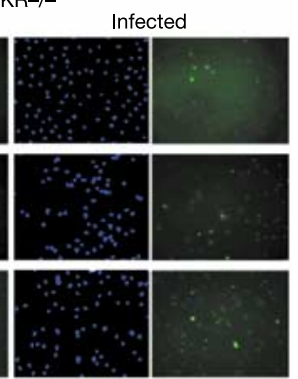

Figure 5 PKR-deficient macrophages are resistant to pathogen-induced apoptosis. a, $\mathrm{PKR}^{+/+}$and $\mathrm{PKR}^{-1-}$ BMDMs were infected with S. typhimurium (SL1344/SipB ${ }^{-}$), S. typhimurium (14028), Y. pseudotuberculosis, or B. anthracis at the indicated multiplicity of infection. Apoptotic TUNEL-positive cells were scored $18 \mathrm{~h}$ post-infection. b. Representative DAPI (blue) and TUNEL (green) staining of $\mathrm{PKR}^{+/+}$and $\mathrm{PKR}^{-/-}$BMDMs
$18 \mathrm{~h}$ post-infection with the indicated pathogens. c, d, wild-type and elF2 $\alpha(S 51 \mathrm{~A})(\mathbf{c})$, or $\mathrm{IRF}^{-1-}$ (d) BMDMs were infected with S. typhimurium (14028) as above and the extent of apoptosis was determined $18 \mathrm{~h}$ later. e, BMDMs from C3H/HeN (T/r4 wild-type) and $\mathrm{C} 3 \mathrm{H} / \mathrm{HeJ}$ (T/r4 mutant) mice were infected with the indicated pathogens and the extent of apoptosis was determined after $18 \mathrm{~h}$. 
MAL, dominate, and exposure of macrophages to bacterial cell wall components, such as LPS, does not result in considerable cell death. However, at least two of the pathogens we examined produce specific virulence factors that inhibit survival pathways ( $\mathrm{p} 38$, $\mathrm{IKK} / \mathrm{NF}-\kappa \mathrm{B})$ and thereby tilt the balance in favour of cell killing.

We identified PKR as an essential component of the TLR4triggered macrophage apoptosis pathway. On the basis of phenotypic and biochemical similarities in the behaviour of PKR- and TRIF- or TRAM-deficient macrophages ${ }^{7,8}$ and the requirement of TRIF for PKR activation, we propose that TLR4 activates PKR and triggers apoptosis through these newly described adaptors. Although PKR is an important contributor to antiviral defences under certain circumstances ${ }^{27}$, its overall contribution to host defences in the case of bacterial infections has not been explored. The results described above suggest that PKR-inhibition may strongly augment macrophage-mediated anti-bacterial responses that do not depend on production of type I IFN and induction of IFN-responsive genes such as iNOS. The decreased expression of iNOS, a major inducer of vasodilation upon PKR inhibition, may also be taken advantage of for prevention of septic shock.

\section{Methods}

\section{Mice and bone-marrow-derived macrophages}

To delete IKK $\beta$ in macrophages, $I k k \beta^{F / F}$ mice ${ }^{12}$ were crossed with MX1-Cre mice (Jackson Laboratory). $\mathrm{PKR}^{-1-}$ mice ${ }^{28}$, IFNRI $^{-1-}$ (A129) and wild-type mice of the same genetic background $(129 / \mathrm{SvEv})^{19}$ were obtained from E. Raz. C3H/HeJ, C3H/HeOuJ, C3H/HeN mice $^{5}$ and bone marrow from lps2 mice $^{7}$ were obtained from B. Beutler. TRAF6 ${ }^{+/-}$,

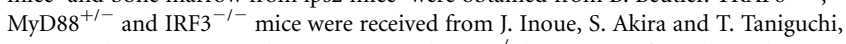
respectively. $\mathrm{C} 57 \mathrm{BL} / 6 \mathrm{~J}$ and $\mathrm{C} 57 \mathrm{BL} / 10 \mathrm{ScCr}\left(\mathrm{TLR}^{-1-}\right.$ ) mice were from the Jackson Laboratory. Unless otherwise mentioned, all knockout mice were of the C57BL/6 background, which is resistant to LT-induced necrosis. BMDMs were prepared and cultured as described ${ }^{4}$.

\section{Analysis of gene expression and cell signalling}

Total cellular RNA was prepared using TRIzol (Invitrogen), quantified by ultraviolet absorption and analysed by real-time polymerase chain reaction (PCR) ${ }^{4}$. Primer sequences are available upon request. All values were normalized to the level of cyclophilin messenger RNA expression. Whole-cell extracts were prepared and PKR activity was measured by autophosphorylation ${ }^{20}$ after immunoprecipitation with anti-PKR antibody (Santa Cruz). PKR recovery was assessed by immunoblotting. Phosphorylation of eIF $2 \alpha$ was detected by immunoblotting with antibody against phosphorylated eIF2 $\alpha$ (Biosource). IKK and MAPK activation were measured as described ${ }^{4}$. Phosphorylation of STAT1 was monitored by immunoblotting with anti-phospho-STAT1 antibody (Cell Signalling).

\section{Bacterial strains, macrophage infections and TUNEL assay}

The wild-type Salmonella typhimurium strains used were SL1344 and 14028.

S. typhimurium $14028 \mathrm{ssaV}$ and $\operatorname{sip} B$ contain mutations in genes that code for components of the SPI2 type III protein secretion system and SipB, respectively. Y. pseudotuberculosi strains YP126 (wild type) and YP26 (YopJ ${ }^{-}$) were obtained from J. Bliska. S. typhimurium BMDM infection was as described ${ }^{29}$, while Y. pseudotuberculosis infection was done as described ${ }^{30}$ with slight modifications: BMDMs were infected with bacteria for $1 \mathrm{~h}$, and then cultured in fresh medium containing gentamicin $\left(20 \mu \mathrm{g} \mathrm{ml}^{-1}\right)$ for another $18 \mathrm{~h}$. The B. anthracis Sterne strain was grown overnight on BHI (brain-heart infusion) agar. A single colony was inoculated into BHI broth or RPMI medium $+10 \%$ FCS (endotoxinfree) in disposable tubes and grown with vigorous shaking to an $\mathrm{OD}_{600}$ of 0.4 . Bacteria were washed with PBS and resuspended in PBS. To prepare heat-killed B. anthracis, bacterial suspensions in PBS were heated to $65^{\circ} \mathrm{C}$ for $30 \mathrm{~min}$. Macrophage cultures were infected as indicated and incubated for $1 \mathrm{~h}$ at $37^{\circ} \mathrm{C}$ in $5 \% \mathrm{CO}_{2} / 95 \%$ air. Gentamicin was added to a final concentration of $20 \mathrm{\mu g} \mathrm{ml}^{-1}$. After $20 \mathrm{~h}$, the medium was removed and cells were fixed with $4 \%$ paraformaldehyde in PBS. TUNEL or DNA fragmentation assays were performed as described ${ }^{4}$.

Received 12 December 2003; accepted 2 February 2004; doi:10.1038/nature02405.

1. Aderem, A. \& Ulevitch, R. J. Toll-like receptors in the induction of the innate immune response. Nature 406, 782-787 (2000)
2. Medzhitov, R. Toll-like receptors and innate immunity. Nature Rev. Immunol 1, 135-145 (2001).

3. Weinrauch, Y. \& Zychlinsky, A. The induction of apoptosis by bacterial pathogens. Annu. Rev. Microbiol. 53, 155-187 (1999).

4. Park, J. M., Greten, F. R., Li, Z. W. \& Karin, M. Macrophage apoptosis by anthrax lethal factor through p38 MAP kinase inhibition. Science 297, 2048-2051 (2002).

5. Poltorak, A. et al. Defective LPS signaling in $\mathrm{C} 3 \mathrm{H} / \mathrm{HeJ}$ and $\mathrm{C} 57 \mathrm{BL} / 10 \mathrm{ScCr}$ mice: mutations in $\mathrm{Tl} 4$ gene. Science 282, 2085-2088 (1998).

6. Medzhitov, R., Preston-Hurlburt, P. \& Janeway, C. A. Jr A human homologue of the Drosophila Toll protein signals activation of adaptive immunity. Nature 388, 394-397 (1997).

7. Hoebe, K. et al. Identification of Lps2 as a key transducer of MyD88-independent TIR signalling. Nature 424, 743-748 (2003)

8. Yamamoto, M. et al. TRAM is specifically involved in the Toll-like receptor 4-mediated MyD88independent signaling pathway. Nature Immunol. 4, 1144-1150 (2003).

9. Kawai, T., Adachi, O., Ogawa, T., Takeda, K. \& Akira, S. Unresponsiveness of MyD88-deficient mice to endotoxin. Immunity 11, 115-122 (1999).

10. Naito, A. et al. Severe osteopetrosis, defective interleukin-1 signalling and lymph node organogenesis in TRAF6-deficient mice. Genes Cells 4, 353-362 (1999).

11. Karin, M. \& Lin, A. NF-kB at the crossroads of life and death. Nature Immunol. 3, 221-227 (2002).

12. Li, Z. W., Omori, S. A., Labuda, T., Karin, M. \& Rickert, R. C. Ikk $\beta$ is required for peripheral B cell survival and proliferation. J. Immunol. 170, 4630-4637 (2003).

13. Kuhn, R., Schwenk, F., Aguet, M. \& Rajewsky, K. Inducible gene targeting in mice. Science 269, 1427-1429 (1995).

14. Horng, T., Barton, G. M. \& Medzhitov, R. TIRAP: an adapter molecule in the Toll signaling pathway. Nature Immunol. 2, 835-841 (2001).

15. Kaufman, R. J. Double-stranded RNA-activated protein kinase mediated virus-induced apoptosis: a new role for an old actor. Proc. Natl Acad. Sci. USA 96, 116935 (1999).

16. Chu, W. M. et al. JNK2 and IKK $\beta$ are required for activating the innate response to viral infection. Immunity 11, 721-731 (1999).

17. Samuel, C. E. Antiviral actions of interferons. Clin. Microbiol. Rev. 14, 778-809 (2001).

18. Lehner, M., Felzmann, T., Clodi, K. \& Holter, W. Type I interferons in combination with bacterial stimuli induce apoptosis of monocyte-derived dendritic cells. Blood 98, 736-742 (2001).

19. Muller, U. et al. Functional role of type I and type II interferons in antiviral defense. Science 264, 1918-1921 (1994).

20. Saelens, X., Kalai, M. \& Vandenabeele, P. Translation inhibition in apoptosis: caspase-dependent PKR activation and eIF2-alpha phosphorylation. J. Biol. Chem. 276, 41620-41628 (2001).

21. Diebold, S. S. et al. Viral infection switches non-plasmacytoid dendritic cells into high interferon producers. Nature 424, 324-328 (2003).

22. Scheuner, D. et al. Translational control is required for the unfolded protein response and in vivo glucose homeostasis. Mol. Cell 7, 1165-1176 (2001).

23. Heylbroeck, C. et al. The IRF-3 transcription factor mediates Sendai virus-induced apoptosis. J. Virol. 74, 3781-3792 (2000)

24. Sato, M. et al. Distinct and essential roles of transcription factors IRF-3 and IRF-7 in response to viruses for IFN- $\alpha / \beta$ gene induction. Immunity 13, 539-548 (2000).

25. Orth, K. et al. Disruption of signaling by Yersinia effector YopJ, a ubiquitin-like protein protease. Science 290, 1594-1597 (2000).

26. Rosenberger, C. M. \& Finlay, B. B. Phagocyte sabotage: disruption of macrophage signalling by bacterial pathogens. Nature Rev. Mol. Cell Biol. 4, 385-396 (2003).

27. Durbin, R. K., Mertz, S. E., Koromilas, A. E. \& Durbin, J. E. PKR protection against intranasal vesicular stomatitis virus infection is mouse strain dependent. Viral Immunol. 15, 41-51 (2002).

28. Yang, Y. L. et al. Deficient signaling in mice devoid of double-stranded RNA-dependent protein kinase. EMBO J. 14, 6095-6106 (1995).

29. Browne, S. H., Lesnick, M. L. \& Guiney, D. G. Genetic requirements for salmonella-induced cytopathology in human monocyte-derived macrophages. Infect. Immun. 70, 7126-7135 (2002).

30. Zhang, Y. \& Bliska, J. B. Role of Toll-like receptor signaling in the apoptotic response of macrophages to Yersinia infection. Infect. Immun. 71, 1513-1519 (2003).

Supplementary Information accompanies the paper on www.nature.com/nature.

Acknowledgements We thank E. Raz, V. Redecke, S. Akira, J. Inoue, T. Taniguchi and B. Beutler for various knockout mice and bone marrow, J. Bliska for Yersiniae strains, N. Sonenberg and R. Medzhitov for gifts of plasmids and antibodies, and M. Delhase for technical assistance. L.-C.H., J.M.P., J.-L.L. and S.M. were supported by postdoctoral fellowships from the Cancer Research Institute, the Irvington Institute, the International Union Against Cancer and the Japanese Society for Promotion of Science, respectively. Work in the laboratories of M.K., D.G.G. and L.E. was supported by grants from the National Institutes of Health. M.K. is an American Cancer Society Research Professor.

Competing interests statement The authors declare that they have no competing financial interests.

Correspondence and requests for materials should be addressed to M.K. (karinoffice@ucsd.edu). 